\title{
Sobre los movimientos alternativos en la actual coyuntura
}

\author{
José María Tortosa \\ Instituto Interuniversitario de Desarrollo Social y Paz, Universidad de Alicante, \\ Alicante, España. \\ Email: jmtortosa3@gmail.com
}

\begin{abstract}
Resumen: Los recientes movimientos sociales (desde las “primaveras árabes” a Occupy Wall Street, pasando por los “indignados” europeos o los estudiantes latinoamericanos) pueden entenderse mejor, sin negar sus componentes locales, si se perciben como alternativas producidas dentro de la lógica del sistema capitalista. A ello se añade la actual coyuntura de inseguridades producidas por la crisis visible desde 2008, que agudiza los motivos de protesta. Ésta, sin embargo, no es mayoritaria, y sus integrantes se plantean -con dificultad- objetivos y medios, al tiempo que oscilan entre la búsqueda de cambios en el sistema y su generalización hacia un cambio de sistema.
\end{abstract}

Palabras clave: Capitalismo, crisis, alternativas, movimientos sociales

\section{On alternative movements in the current conjuncture}

Abstract: Recent social movements (from «Arab spring» to Occupy Wall Street, passing through «the outraged» European or the Latin American students) can be understood better, without denying local components, if alternatives are perceived as produced within the logic of the capitalist system. To that one must add the current conjuncture of insecurities produced by the visible crisis since 2008, which exacerbates the motives for protest. This, however, is not dominant, and its members pose -with difficulty- goals and means, while they oscillate from seeking changes within the system and its generalization towards a system change.

Key words: Capitalism, crisis, alternatives, social movements

\section{Sobre os movimentos alternativos na atual cojuntura}

Resumo: Os recentes movimentos sociais (da "primavera árabe” a Ocupar Wall Street passando por os "indignados” europeus ou os estudantes latino-americanos) pode ser entendido melhor, sem negar seus componentes locais, se as alternativas são percebidas como produzidas dentro da lógica do sistema capitalista. Esta situação é agravada pela actual clima de insegurança produzida pela crise visível desde 2008 que agravam injustiças. Isto, no entanto, não é dominante e os seus membros se confrontam com difícultade metas e meios, enquanto o intervalo de pesquisa de mudanças no sistema e sua generalização a uma mudança do sistema.

Palavras-chave: capitalismo, crise, alternativas movimentos sociais 
[C]onsciousness about ambiguity may help us overcome the tyranny of false dichotomies. Neither peace nor health should be taken for granted -if they are, they are soon lost- but be seen as something to work on every day, one way or the other.

Johan Galtung, "The Bad in the Good and the Good in the Bad",

Noviembre 2011.

That the Occupy movement is unprecedented seems appropriate because this is an unprecedented era, not just at this moment but since the 1970s. Noam Chomsky, “Occupy the Future”, Noviembre 2011.

Las alternativas que se producen en la particular inestabilidad del sistema mundial actual, sea o no coyuntural, parece que se comprenden mejor si, en primer lugar, se las sitúa dentro de lo que es la lógica de dicho sistema, sus reglas del juego, ya que no sería de descartar que, una vez más, dichas alternativas fuesen fagocitadas por la aplastante capacidad del sistema de incorporarlo todo, en especial los cuerpos extraños.

Sin embargo, es obvio que algunas situaciones de aparente estabilidad se vinieron abajo (hay muy pocas cosas humanas irreversibles exceptuando la muerte), razón por la que no habría que excluir que eso pudiese producirse ahora, del mismo modo que, como se ha dicho, no hay que excluir (la historia lo sugiere) que la capacidad de recuperación del sistema volviese a dar señales de su fuerza.

Por otro lado, hay que insistir, la situación que atraviesa el sistema mundial, al decir de muchos de sus observadores, no tiene precedentes. Cierto que se puede pensar en la crisis cíclica de 1929 con la que, en efecto, guarda resonancias (la historia no se repite ni en tragedia ni en comedia, pero sí hay etapas que "resuenan" unas con otras y, por tanto, se puede aprender de ellas por lo menos para hacerse preguntas). La crisis económica, el descrédito de la democracia, el auge de los movimientos totalitarios cargados de verdades absolutas e intolerantes de cualquier duda y la potencia hegemónica en crisis son elementos a tener ahora en cuenta como se pudieron tener en cuenta entonces incluso por parte de los movimientos alternativos (como los Wandervögel) que terminaron fagocitados por el nazismo. Pero es obvio que no estamos en las mismas: en el 29 no había conciencia de la huella ecológica que dejaba en el Planeta la especie en general y los países enriquecidos en particular y no se discutía el acceso al petróleo abundante y barato aunque el Big Game previo ya se había encargado de repartir su acceso y distribución. Ni había 1.000 millones de personas hambrientas en el mundo.

Es en ese doble contexto (las reglas del juego por un lado como factor constante y la coyuntura actual por otro como precipitante) en el que se sitúa la oleada de muy variados "indignados" entre los que ha podido surtir efecto el contagio de unos a otros ${ }^{1}$. El movimiento no comenzó por 
algunos de ellos aunque sus participantes lo crean. Cierto que no comenzó con "Occupy Wall Street" aunque su impacto mediático, al provenir del país todavía hegemónico, haya sido mayor. Pero todos se sitúan en una coyuntura que es preciso tener en cuenta, al igual que hay que tener en cuenta sus antecedentes. También es preciso sacar lecciones sobre cuáles son sus desafíos y posibilidades.

Ese es el esquema del presente trabajo: las reglas del juego como razón última, la coyuntura como precipitante, la sucesión de movimientos y las lecciones que pueden sacarse para el futuro.

\section{La razón última: la lógica del sistema}

The protesters are dismissed as dreamers, but the true dreamers are those who think that things can go on indefinitely the way they are, just with some cosmetic changes.

Slavoj Zizek, “The Violent Silence of a New Beginning”, Octubre 2011.

Social protest has found fertile ground everywhere: a sense that the "system" has failed, and the conviction that even in a democracy, the electoral process will not set things right - at least not without strong

pressure from the street.

Joseph Stiglitz, “The Globalization of Protest”, Noviembre 2011

Un texto (“Del capitalismo como 'sistema parásito’ ”), publicado por Zygmunt Bauman en 2009 en Clarín $^{2}$ recuperaba algunas ideas de Rosa Luxemburg de las que, probablemente, también bebió Immanuel Wallerstein: el capitalismo realmente existente funciona mediante la "colonización” de "territorios vírgenes” para la explotación. Esta colonización puede ser física (conquista, ocupación, “descubrimientos” varios), puede ser comercial (el neocolonialismo inaugurado por los Estados Unidos al sustituir a Inglaterra como potencia hegemónica colonial) o puede ser financiera. En este último caso, el sistema actual se ha mantenido, indica Bauman, pasando del capitalismo del que hablaba Max Weber (austero, ahorrador, calculador, capitalismo inversor a partir de la fórmula $\mathrm{S}=\mathrm{I}$, ahorro igual a inversión) al capitalismo de consumo y, de éste, al capitalismo del crédito que es el que habría entrado en crisis como todos los modos anteriores de mantener en vigor la norma básica del sistema, que es la del beneficio sin límites físicos, sociales y económicos o, si se prefiere, la de la acumulación incesante de capital. Cierto que también intentó colonizar el espacio virtual o ciberespacio ${ }^{3}$ (proceso del que formaría parte la burbuja del punto.com), pero siempre mediante burbujas que reventaban (es lo único que se sabe cierto sobre las burbujas: que revientan, como sucedió con la burbuja inmobiliaria en zonas del sistema -como el Japón en los años 90- y que ahora ha contaminado a todo el planeta con las subprime estadounidenses, con particulares efectos en las Españas, que tenía la propia). 
A pesar del corto espacio de tiempo que ocupa (en realidad, menos del "corto siglo XX” del que escribe Eric Hobsbawm) la Guerra Fría tiene particular interés para el tema del presente trabajo dada su aparente estabilidad y previsible perdurabilidad. Por ambas partes del conflicto se daba por supuesto que se terminaría cuando, en un futuro tal vez lejano, una de las partes suprimiera a la otra o, por lo menos, la sobreviviera (así lo afirmó Kruschev y reprodujo Time), pero por debajo de esa retórica es posible que lo que subyaciese fuera el convencimiento (humano, demasiado humano) de la más absoluta estabilidad, sobre todo por el esquema MAD (loco, en inglés, pero acrónimo de Destrucción Mutua Asegurada mediante el arma nuclear en ambos contendientes). No es de extrañar que el fin de aquella aparente estabilidad asegurada por las armas fuese saludada por publicaciones como El fin de la Historia, versión hegeliana que podría ser marxista, en la que la Historia acaba cuando, sean ideas o clases sociales, una de las dos partes en liza (porque solo pueden ser dos) desaparece o es vencida por la otra. Sin embargo, pocos fueron los que escribieron, como Andrei Amalrik en 1979, un “¿Podrá la Unión Soviética sobrevivir a 1984? (Will the Soviet Union Survive Until 1984?). Lo más frecuente era dar por supuesto que la Unión Soviética seguiría existiendo como ahora se da por supuesto que los Estados Unidos seguirán eternamente como potencia hegemónica cuando, si otros cayeron, dicho país también podría dejar de serlo.

Vista en perspectiva, la Guerra Fría no tuvo momentos de riesgo para el mantenimiento del sistema, a pesar de la retórica en contra, dado el acuerdo tácito y no tan tácito (de Yalta a Malta) entre ambas potencias de mantener la división del mundo de modo que cada cual tuviese sus propias “colonias” sobre las que se ejercía el poder colonial de modo clásico aunque con algunas variantes. La más evidente era cómo se aplicaba, en un bloque y otro, el "intercambio desigual” entre materias primas y productos manufacturados: mientras Rusia mantenía un fuerte peso de las materias primas en sus exportaciones, importando productos manufacturados de sus satélites y debilitándose consiguientemente, los Estados Unidos hacían lo contrario y eran criticados por los economistas marxistas que, sin embargo, no lo detectaban en el bando soviético.

En la peor de las hipótesis, el enfrentamiento necesario, sobre todo por el papel keynesiano (invertido y pervertido, pero keynesiano) que tenía y tiene el sector del armamento en los Estados Unidos, se producía mediante contendientes interpuestos en las llamadas "guerras de baja intensidad”. Si hubiese habido algún riesgo extremo, se habría producido, en tiempos de John F. Kennedy y Nikita Kruschev, en la "crisis de los misiles" en la que pudo haberse llegado a una confrontación con escalada nuclear y, por tanto, con riesgo de "invierno nuclear", pero que se solucionó con un "tit-for-tat": retirada del reciente despliegue estadounidense en Turquía y no instalación de los misiles soviéticos en Cuba, cosa que cada contendiente anunció como triunfo propio mientras silenciaba la parte correspondiente al otro bando. 
Pues bien, lo que ahora mantiene vivo al sistema es la colonización... del futuro: el beneficio de hoy se obtiene cargando sobre las espaldas no de los "salvajes" o de los "primitivos pre-capitalistas" como comenzó el sistema-mundo contemporáneo, sino sobre las generaciones futuras. Eso es, al fin y al cabo, la actual crisis de la deuda que, para gestionarla, exige rigor presupuestario por parte de los Estados que han corrido en socorro del vencedor.

En este sistema, dado que funciona mediante la desigualdad, como ya reconocía Adam Smith a propósito del mercado que se nutre de la misma al tiempo que la genera, produce lo que en un primer momento fue visto como "sus propios sepultureros", los proletarios, como se decía en el $\mathrm{Ma}$ nifiesto Comunista, aunque los previstos por Marx y Engels no lo consiguieron como tampoco lo consiguieron los indignados por los efectos del maquinismo llamados luditas por la localidad inglesa (Ludd) en la que comenzaron las protestas por la introducción de lo que ahora se llamarían "nuevas tecnologías", que ha sido otra de las características del sistema: la de buscar cíclicamente, como mostró Schumpeter, nuevos instrumentos técnicos para obtener el beneficio.

En cualquier caso, en el sistema se producen reacciones contra esa desigualdad considerada injusta por los perdedores y a favor de estos que son los que pierden en el intercambio colonial que incluye el "colonialismo interior" no solo espacial sino, sobre todo, social: la lucha de clases de "los de arriba" contra "los de abajo", que es el fenómeno recurrente en el sistema. "Los de abajo", que es terminología que usa George Orwell en su Mil novecientos ochenta y cuatro, o el "99 \%" que es terminología contemporánea, de vez en cuando hacen oír su voz reivindicando derechos, conquistas anteriores mermadas o proyectos alternativos de futuro. No siempre han alcanzado una escala mundial ya que, en contra de lo dicho en el Manifiesto, los proletarios sí que tienen patria ya que, de hecho, el nacionalismo (estatal o subestatal, raramente supra-estatal como se pretendió con el europeísmo o se pretende con el latinoamericanismo) es la única ideología dominante a escala mundial. Sin embargo, son frecuentes, por parte de los involucrados en los actuales movimientos alternativos, los pronunciamientos sobre la fuerza que estos están teniendo en sus diversas versiones (Foro Social Mundial incluido) que corren parejo con los anuncios de una "crisis terminal del sistema".

¿Se acaba con eso el capitalismo y su lógica? No está tan claro. En primer lugar, porque no es tan evidente que la lógica del beneficio sin límites haya sido privativo de esta economía-mundo. Pero, con independencia de las discusiones de escuela que enfrentaron a Andre Gunder Frank defendiendo la no especificidad del capitalismo a este respecto y, por otro lado, a Immanuel Wallerstein y Samir Amin afirmando lo contrario, el caso es que cuando se vio que ya no había territorios vírgenes (territorios físicos) que colonizar, se pensó que el capitalismo entraba en "crisis terminal" como sucedió en tiempos de la Revolución de Octubre que se llevó por delante, físicamente, a Nikolái Kondrátiev por afirmar que se trataba de una "crisis cíclica" para nada "terminal” como predicaba la línea oficial del 
Partido. De momento, el sistema ha sido capaz de superar las sucesivas “etapas superiores del capitalismo”. En el caso anterior, mediante la invención del Estado de Bienestar reductor de las contradicciones entre "los de arriba” y "los de abajo”. En el actual, mediante la aplicación del Estado de Bienestar para ricos y la colonización del futuro (un país que debe -entre público y privado- tres veces su PIB, tiene que colonizar su futuro y aguantar mediante rigor presupuestario mientras pueda y no se invente otra cosa), fenómenos acompañados por una inmensa manipulación informativa que, como indicaba Howard Zinn, es eficaz en condiciones de desconocimiento de la historia y en las de exceso del nacionalismo de "my country, right or wrong”. La capacidad de afrontar las crisis creadas por el mismo sistema (no por los anti-sistema, ciertamente) es proverbial.

\section{Un precipitante: las inseguridades actuales}

They were so puzzled that they started scratching their heads, but that did no good, even when they all did it together and called it an International Conference. The plain fact is that governing classes of the world are too ignorant and stupid to be able to think through such a problem and too conceited to ask advice of those who might help them.

Bertrand Russell, “The Modern Midas”, 1932

The biggest question in America these days is how to revive the economy. The biggest question among activists now occupying Wall

Street and dozens of other cities is how to strike back against the nation's almost unprecedented concentration of income, wealth, and

political power in the top 1 percent. The two questions are related (...). Here, as elsewhere, people are outraged at what feels like a rigged game - an economy that won't respond, a democracy that won't listen, and a financial sector that holds all the cards. Here, as elsewhere, the people are rising.

Robert Reich, “Washington Pre-Occupied”, Noviembre 2011

La lógica del sistema, sus cambiantes reglas del juego bajo el principio básico del beneficio sin límite físico, social o económico, ha tenido efectos devastadores en la actualidad. Está, antes de todo, la crisis económica que afecta, de momento, a los países centrales en los que un decrecimiento o crecimiento bajo se traduce en reducción de empleo y aumento de problemas que lo acompañan después de que los gobiernos corriesen a salvar a los bancos con problemas y, con ello, entraran en déficit o lo aumentaran. Posteriormente, su necesidad de endeudamiento se fue solventando con préstamos para afrontar los gastos corrientes no la inversión productiva, reduciendo así el gasto público y, por tanto, introduciendo un nuevo factor de desaceleración. 
No es la primera vez que sucede tal cosa aunque probablemente lo sucedido en otras ocasiones ha sido más local o no afectó de la misma forma a los países centrales. Precisamente, a diferencia de la crisis del 29, lo que ahora se produce es una acumulación de problemas nuevos que se añaden, interactuando, a los económicos, generando así una particular inestabilidad del sistema en su conjunto, comenzando por su potencia hegemónica, los Estados Unidos ${ }^{4}$.

En primer lugar, están los problemas medioambientales: además se colonizar el futuro, parece claro que la colonización de la Naturaleza ha alcanzado niveles cuya "huella ecológica” podría alcanzar niveles de difícil recuperación homeostática. Es cierto que se puede seguir discutiendo, a veces por motivaciones espurias, si el Planeta se encuentra ante la amenaza (o la realidad) de un cambio climático. El hecho es que aparece ya como vocabulario del Banco Mundial (Informe sobre el desarrollo mundial 2010: Desarrollo y cambio climático), Agencia Internacional de la Energía (World Energy Outlook 2011) y hasta en el informe de Transparencia Internacional (Informe Global de la Corrupción 2011: cambio climático), pasando por los escenarios que plantea la CIA en su cuatrienal ejercicio de prospectiva (Global Trends 2025, publicado en 2008: escenario 2 y escenario 4).

Lo que ya no es discutible, sobre todo después del informe de la Universidad de Berkeley ${ }^{5}$, es el hecho del calentamiento global, vaya éste a seguir o no. Sus efectos hasta ahora sobre sequías, inundaciones y malas cosechas han sido suficientemente constatados. Si la especie humana está en riesgo de crisis profunda es pronto para saberlo, pero sí es notable que gobiernos y ciudadanos están dispuestos (y las encuestas internacionales son explícitas al respecto) a sacrificar el medioambiente con tal de volver a tasas de crecimiento económico que permitan el retorno del empleo. Suicidas.

El World Energy Outlook publicado por la Agencia Internacional de la Energía en 2011 reconocía dos hechos relevantes para la presente discusión: por un lado, la inminencia (si no presencia) del llamado "pico del petróleo", momento en que el consumo ya se hace a costa de las reservas sin que los nuevos descubrimientos (en Argentina, Brasil o País Vasco por poner ejemplos) sean suficientes para compensar el agotamiento de los recursos aunque aparentemente el consumo siga aumentando. Por otro lado, el informe reconoce el impacto que el consumo de combustibles fósiles tiene en el calentamiento y eventual cambio climático.

Junto a esta crisis energética se presenta también, aunque hay motivos para desconfiar de algunos planteamientos de organismos internacionales (como se pudo desconfiar de las alarmas de la Organización Mundial de la Salud a propósito de algunas gripes como la aviaria o la porcina H1N1-), una crisis alimentaria que, como las anteriores, no afecta por igual a todas las partes del mundo. Si la crisis económica y la energética son problemas sobre todo de los países centrales (enriquecidos, "desarrollados”, del Norte), los efectos de la crisis medioambiental se notan más, de momento, en los países periféricos y, ciertamente, la crisis alimentaria, sea 
o no fabricada, les afecta igualmente a través de hambrunas complejas en las que se mezclan diversos factores, pero en las que el elemento alimentario es una absoluta obviedad. No es descabellado pensar en mil millones de subnutridos (hambrientos) en el mundo en la actualidad, con una pequeña incidencia en los países centrales (en torno a los 19 millones), pero que aparece en proporciones enormes en algunas de las constatables catástrofes humanitarias actuales y que, en general, todo hace pensar que seguirá creciendo haciendo así imposible el logro del correspondiente Objetivo de Desarrollo del Milenio, ya de por sí planteado en los términos que hacían más fácil su logro, es decir, usando porcentajes y no cifras absolutas. Sucede lo mismo con el ODM referido a la pobreza: se puede reducir el porcentaje de pobres (definidos como quien dispone de menos de 1,25 dólares al día, que ya es cálculo problemático) y el de hambrientos, sin cambiar su cuantía, simplemente aumentando el resto de personas no clasificadas en tal categoría.

El resultado de esta acumulación de crisis es el sentimiento de inseguridad que se difunde entre amplias capas de la población. Tal vez no exactamente el "99 \%", pero sí en sectores medios y medio-bajos de los países centrales. Estas inseguridades incluyen, pues, la inseguridad económica (el futuro del propio empleo y de la propia pensión ${ }^{6}$, pero que solo es una parte del problema) y la inseguridad energética (los efectos del "pico del petróleo” y la necesaria transición energética para la que no hay muchos mapas $^{7}$ ), cosa que afecta, sobre todo, a los países hasta ahora centrales, pero no por ello eternamente centrales ${ }^{8}$. Sin embargo, no afecta tanto a los países periféricos que reciben el embate de la inseguridad alimentaria y la medioambiental (las catástrofes “naturales” son clasistas: afectan más a las periferias y, sobre todo, a las periferias de los países periféricos).

Y todo ello en un ambiente generalizado de descrédito de las democracias realmente existentes y de sus respectivas “clases gobernantes” como las llamaba Bertrand Russell en 1932, en plena crisis del 29, en términos que podrían ser contemporáneos. El descrédito de la clase política va unido a una más que evidente crisis de confianza ${ }^{9}$ en muchas instituciones, perceptible en amplias capas de la población y, entre dichas instituciones, están no solo los partidos sino también los sindicatos, las iglesias...

El ejemplo para el conjunto español que se presenta no es excepcional. Los datos son del Barómetro del Centro de Investigaciones Sociológicas de octubre de $2011^{10}$ y la respuesta se refiere al grado de confianza que le merecen al entrevistado las instituciones que se le presentan, dándole la opción entre 0 (ninguna confianza) y 10 (máxima confianza). Aceptando el símil escolar, únicamente “aprueban” las fuerzas armadas y, subiendo décimas, los medios de comunicación y la monarquía, aunque en este último caso, junto al de la Iglesia Católica, la desviación típica de las respuestas da los valores más altos, indicando, probablemente, una mayor polazación de confianzas y desconfianzas, cosa que no sucede con los partidos políticos, que son los que menos confianza despiertan entre los entrevistados. 


\begin{tabular}{|l|r|r|c}
\hline & Media & Sigma & $(\mathrm{N})$ \\
\hline Partidos politicos & 2,76 & 2,51 & $(2.392)$ \\
\hline Gobierno & 3,24 & 2,75 & $(2.419)$ \\
\hline Parlamento & 3,52 & 2,67 & $(2.266)$ \\
\hline Iglesia Católica & 3,66 & 3,18 & $(2.386)$ \\
\hline Parlamento de su C.A. & 3,88 & 2,80 & $(2.230)$ \\
\hline Gobierno de su C.A. & 3,93 & 2,83 & $(2.354)$ \\
\hline Consejo G. Poder Judicial & 3,94 & 2,71 & $(2.024)$ \\
\hline Tribunal Constitucional & 4,07 & 2,75 & $(2.057)$ \\
\hline Defensor del Pueblo & 4,55 & 2,77 & $(2.069)$ \\
\hline Monarquia & 4,89 & 3,21 & $(2.388)$ \\
\hline Medios comunicación & 4,97 & 2,47 & $(2.385)$ \\
\hline Fuerzas Armadas & 5,65 & 2,95 & $(2.349)$
\end{tabular}

Otras encuestas internacionales dejan a la clase política en puestos igualmente bajos en cuanto a su aceptación o la valoración general que provocan en sus respectivos ciudadanos aunque con diferencias de país a país según su historia, cultura política dominante y su progreso o retroceso en la satisfacción de las necesidades básicas de sus ciudadanos ${ }^{11}$. La contradicción que subyace es la de cómo conseguir, sin cambiar las reglas del juego, crecer para crear empleo y, al mismo tiempo, decrecer para responder a los desafíos medioambientales cuando optando por el primer término tampoco se consigue el empleo, con lo que los males se acumulan. Y cómo hacerlo desde la política después de una larga etapa en la que el mantra, ahora ya prácticamente inexistente, ha sido "menos Estado, más mercado" que ha producido y acumulado desregulaciones, sobre todo en el sector financiero y ha estado entre los factores que han llevado a la actual crisis económica que primero fue financiera, pero que, en todo caso, ha sometido a los gobiernos a los intereses financieros mundiales ${ }^{12}$.

\section{Diez respuestas recientes}

Quien piensa sabe decir no y esa palabra constituye una revolución, pero ese no tiene un sentido cuando se trata de un no colectivo, de una voluntad colectiva. No obstante, todos sabemos que también el no se corrompe, se acomoda y se convierte poco a poco en un sí. Cuando eso ocurre, no hay más remedio que volver a decir otra vez no. José Saramago, En sus palabras, 2010.

"We are the 99 percent" is a great slogan. It correctly defines the issue as being the middle class versus the elite (as opposed to the middle class versus the poor). And it also gets past the common but wrong establishment notion that rising inequality is mainly about the well educated doing better than the less educated; the big winners in this new Gilded 
Age have been a handful of very wealthy people, not college graduates in general. Paul Krugman, "We Are the 99.9\%”, Noviembre 2011

Occupy Wall Street and its allied movements around the country are more than a walk in the park. They are most likely the start of a new era in America. Historians have noted that American politics moves in long swings. We are at the end of the 30-year Reagan era, a period that has culminated in soaring income for the top 1 percent and crushing unemployment or income stagnation for much of the rest. The overarching challenge of the coming years is to restore prosperity and power for the 99 percent.

Jeffrey D. Sachs, “The New Progressive Movement”, Noviembre 2011.

Las reacciones ante estos hechos no han tenido una fuerte correlación con los mismos ni siquiera con la evolución de la conciencia de ellos. Tampoco han asumido las mismas formas en países centrales que en países periféricos, aunque lo habitual es que las reacciones de los países centrales reciban mayor apoyo mediático que acabe haciendo creer a sus participantes que se encuentran en un movimiento “adamítico”, de creación de un mundo nuevo sin antecedente alguno, que no siempre se les puede atribuir en buen empirismo.

En junio de 1932, en plena crisis, unos 20.000 veteranos de guerra acamparon en Washington, a la vista del Capitolio, protestando por su imposibilidad de encontrar trabajo, exigiendo el pago inmediato, no diferido, de la indemnización debida (se llamó la "Bonus Army”) y criticando los rescates bancarios multimillonarios y ayudas a las grandes empresas (la Reconstruction Finance Corporation) para los que sí había fondos y que habían sido aprobados por el Congreso. En aquellos tiempos, radio-predicadores como el sacerdote católico Charles Coughlin (por cierto, antijudío), tronaban contra "la codicia de banqueros y financieros" y criticaban a un gobierno que "si tiene para pagar 2.000 millones a los banqueros (...), no se ve por qué no tiene 2.000 millones para pagar a los soldados”13.

La perspectiva temporal podría ampliarse hasta la Primavera de los Pueblos en la Europa de 1848 con ramificaciones en una cincuentena de países desafiando a monarcas y autócratas mientras Marx y Engels terminaban el Manifiesto Comunista. Pero parece que es suficiente remontarse a algo más de un siglo después: 1968.

En mayo de 1968, en el cambio de ciclo económico largo de una fase ascendente a una descendente, el movimiento fue mucho más general aunque básicamente de estudiantes y con desconfianza por parte de muchos sindicatos. Se trataba del "seamos realistas, pidamos lo imposible" y encontraron la arena debajo de los adoquines parisinos aunque con ramifi- 
caciones en numerosos países no solo centrales, con componentes como la ofensiva del Tet en Vietnam, el movimiento contra la guerra en muchas partes del mundo, la Primavera de Praga o la masacre en la plaza de Tlatelolco en México Distrito Federal.

La evaluación del movimiento es ambigua. Para algunos, como Immanuel Wallerstein, se trató de una "revolución mundial”, con causas comunes (el agotamiento de un modelo de Estado) y con efectos perdurables en la percepción de los problemas. Para otros, no tuvo mayores efectos, como pudo verse en los siguientes pasos políticos en Francia (referéndum y elecciones) y se ha visto con la evolución de algunos de sus líderes.

No son los únicos "indignados" que en el mundo han sido. La lista incluiría desde fenómenos de trascendencia local (como el Bonus Army) hasta los que han tenido impacto mundial (y los que, explícitamente, se proponen como movimientos que buscan "ocupar el mundo"). Esta es una lista (incompleta) de los movimientos locales recientes con algunas observaciones sobre los mismos que pueden servir de advertencia para los movimientos que quieren ser mundiales, que todavía son muy heterogéneos y que, por tanto, carecen de una táctica común y, muchos más, de una estrategia común.

\begin{tabular}{|ll|l|}
\hline \multicolumn{2}{|l|}{ Movimiento } & \multicolumn{1}{c|}{ Observaciones } \\
\hline 1. & Piqueteros & Omnipotencia de ideas \\
2. & Forajidos & Evaluación al tiempo \\
3. & Islandeses & Tamaño y tradición \\
4. & Primaveras árabes & Importancia obstáculos \\
5. & Grecia/Portugal & Frustración y agresividad \\
6. & Estudiantes Chile & Maximalismo \\
7. & Indignados Israel & Lo que no se discute \\
8. & Tottenham & Solo expresión \\
9. & TIPNIS & Instrumentación \\
\hline
\end{tabular}

1. Se traen a colación los piqueteros argentinos (en particular, el movimiento iniciado en 1993) porque sirve de advertencia fundamental: su eslogan más conocido ("Que se vayan todos”) fue expresión de un deseo, pero no puso en funcionamiento mecanismos que permitieran su cumplimiento. De hecho, "se quedaron todos".

2. En abril de 2005, “forajido" fue el nombre que, en el Ecuador, el presidente Gutiérrez dio a los que se manifestaban contra su presidencia, nombre que fue asumido por los manifestantes que acabaron iniciando la "revolución ciudadana” que daría pie al triunfo de Rafael Correa. La evaluación inmediata, en el mismo mes de abril, que hacía Alberto Acosta, dirigente del que sería el movimiento y después partido de Rafael Correa y presidente que fue de la Asamblea Constituyente, concluía diciendo que "ya se sienten vientos de cambio que serán determinantes para la vida de 
este pequeño país andino y que, quizás, también sirvan de ejemplo en la región”. Tenía razón. Pero el caso ecuatoriano, como el de tantas revoluciones (incluida la Gran Revolución -la francesa- , la Revolución Mexicana y la Revolución de Octubre), muestra que la evaluación no puede hacerse ni en el momento de su inicio ni, mucho menos, en el de su triunfo, sino con perspectiva temporal como para saber si ha habido o no una "revolución ciudadana”. El mismo Acosta, ya en 2011, dirá: "Uno de los puntos más débiles del presidente Correa es que es un Gobierno que presume de Revolución Ciudadana y lo que es notorio es el déficit de participación ciudadana. El déficit de ciudadanía en la Revolución Ciudadana es inocultable”.

3. El caso islandés ${ }^{14}$, ya en 2011, muestra la importancia de la historia reciente (en su caso, de las luchas por la independencia lograda en 1918 como reino y en 1944 como república), de las tradiciones culturales y de lucha y de la población relativamente reducida (algo más de 300.000 habitantes). La onda de protestas que se producen en el mundo, reacción ante el sistema precipitada por las condiciones inmediatas de inseguridad e insatisfacción, se "refracta” en cada circunstancia local de modo que no es tan sencillo exportar o importar métodos y estrategias. Con otra historia, cultura política y condicionamientos es difícil ser "Islandia” con independencia de qué se haya conseguido realmente con las reacciones populares como sucedió con los piqueteros.

4. Las “primaveras árabes” son sumamente heterogéneas ${ }^{15}$. En efecto, no es lo mismo, por citar a sus comienzos, Túnez que Egipto y, después, no sería lo mismo Bahrein, intervenida por Arabia Saudita, y Libia, intervenida por la OTAN bajo mando estadounidense. La advertencia, en este caso, se refiere al conocimiento de los posibles obstáculos que los levantamientos populares pueden encontrar y que, de nuevo, difieren de un lugar a otro y, por tanto, producirán efectos diferentes, además del ya indicado en el caso del Ecuador de la necesidad de ver la evolución de dichos efectos a lo largo del tiempo. No se excluye, aplicada a Egipto, la advertencia que se deriva del dicho de Tancredi en El Gatopardo: "Se vogliamo che tutto rimanga come è, bisogna che tutto cambi".

5. Las reacciones en Portugal y, sobre todo en Grecia, a los sucesivos "planes de ajuste" y "terapias de choque” impuestas desde el exterior recuerdan lo que, en su momento, se llamaron "IMF riots", las acciones de protesta de una población que ve disminuir sus niveles de vida por causa de dichos “planes” o “terapias”. Tienen elementos en común, en este sentido, con lo sucedido entre los años 70 y 80 en América Latina, pero también los tienen con algunos de los países árabes en los que ha habido "primavera”. Hay, sobre todo, emoción y comportamiento expresivo de la misma, que suele ir dirigida contra el gobierno que ha aceptado velis nolis la imposición exterior y no contra el organismo, institución o personas que la han impuesto. De hecho, de las 16 elecciones generales producidas en Europa después del 2007, en solo 4 el gobierno ha logrado ser reelegido, además de los casos extremos de soslayo de las reglas democráticas como han sido 
Grecia e Italia. “Ira, desdén y sensación de impotencia” titulaba un periódico de Madrid la reacción popular ante la "agónica negociación entre partidos para buscar una salida a la crisis" que "fomenta la frustración y el disgusto de los griegos hacia la clase política”. Sentimientos, sí.

6. El caso de los estudiantes chilenos implica también una advertencia: en una negociación, como la que podría haber sido la suya con su gobierno, es aconsejable llegar con peticiones ligeramente superiores a lo que se pretende, para así tener un margen de negociación; si, en cambio, se llega pidiendo exactamente lo que no se está dispuesto a renunciar, el maximalismo de ambas partes puede llevar a un punto muerto. Mucho más problemático sería si, como en mayo del 68, se entona el "seamos realistas, pidamos lo imposible”. De momento, las manifestaciones por una educación de calidad se produjeron, el 24 de noviembre de 2011, en Argentina, Brasil, Chile, Colombia, Costa Rica, Ecuador, Guatemala, Honduras, México, Paraguay, Perú, Uruguay y Venezuela ${ }^{16}$. No es, por tanto, un movimiento de tono menor o puramente local o localista.

7. Los “indignados” de Israel lo son por determinadas condiciones de vida que encuentran inaceptables, en particular las que se refieren al acceso a una vivienda digna. Comenzó en julio de 2011 a propósito del precio de determinados alimentos y acabó extendiéndose a plantear la cuestión de la desigualdad social en el país. Conviene, en este caso (y en los demás), levantar acta, además de sus reivindicaciones, también de sus silencios o, si se prefiere, de lo que queda fuera de la agenda de los "indignados”, aunque no sea más que por no alienarse sectores importantes de la propia sociedad. Porque se puede estar en contra de determinadas políticas de un gobierno (como lo estaba la "Bonus Army") sin por ello poner en cuestión otras políticas del mismo gobierno de tipo imperialista y, aquí, del tipo expansionista con viviendas en los nuevos asentamientos. De hecho, se ha pasado como gato sobre brasas respecto a la situación de Cisjordania y las prácticas israelíes allí.

8. Dos reacciones populares más, ambas muy locales y significativas, pero, al mismo tiempo, muy diferentes entre sí. Por un lado, los disturbios iniciados en Inglaterra, en concreto en Tottenham, puramente expresivos del descontento y muy parecidos a los de los "banlieusards" parisinos de 2005. No había ningún objetivo a alcanzar: solo expresión de rabia y frustración. La sucesión de huelgas en el Reino Unido el 30 de noviembre de 2011, en cambio, tuvieron un tono más convencional ${ }^{17}$.

9. En el otro extremo, la "larga marcha” en Bolivia de los indígenas contrarios al trazado de una carretera que atravesaría su territorio y parque natural (TIPNIS), sin haberles consultado como pide la Constitución del Estado Plurinacional. Objetivo concreto, medios concretos -con mucho esfuerzo y sacrificio- y éxito inmediato al dar marcha atrás el gobierno y la multinacional brasileña que iba a construir la ruta. La advertencia, aquí, es otra: la necesidad de distinguir entre los motivos o las causas de una reacción por un lado y, por otro, las posibilidades de manipulación que pueden 
proporcionar y que no se convierten en una negación de dichas causas. Fuesen o no fuesen manipulados contra el gobierno del presidente Morales, el hecho es que los indígenas tenían razón, aunque, como en el caso ecuatoriano de los “forajidos”, habrá que ver qué sucede en el tiempo y si la "ley corta” abortando la carretera es sucedida por prácticas que la favorecen. A este caso del TIPNIS hay que añadir que ha habido numerosos casos semejantes en el Ecuador contemporáneo de oposición a prácticas extractivistas y desdeñosas con los pueblos originarios, pero han sido de menor impacto social por haber tenido menos participantes y menor impacto mediático aunque, en algunos casos, tuvieron una respuesta violenta por parte gubernamental mayor que la producida en un determinado momento con los “marchistas” del TIPNIS boliviano.

10. El movimiento del 15-M y sus variantes locales y su evolución hacia el 15-O a través de los diversos “Occupy” (Wall Street inicialmente, un 17-S, pero después en más ciudades con la pretensión de “internacionalizarse”) están en otra dimensión a pesar de su evidente parentesco con muchos de los que aparecen en la tabla. Joseph Stiglitz titulaba un artículo con "La globalización de la protesta” en el que se refería a que "El movimiento de protesta que nació en enero en Túnez, para luego extenderse a Egipto y de allí a España, ya es global: la marea de protestas llegó a Wall Street y a diversas ciudades de Estados Unidos” y comentaba, casi como el padre Coughlin en 1932, "Tenemos un sistema donde a los banqueros se los rescató, y a sus víctimas se les abandonó”.

Es, sin duda, pronto para hacer, y menos desde fuera, una evaluación de estos movimientos sobre todo si el $15-0$ se consolida ${ }^{18}$ y perdura, cosa que está por ver ${ }^{19}$ como está por ver si las indignaciones locales, muchas veces con planteamientos no solo locales sino incluso localistas, se articulan en respuestas colectivas a escala mundial sin el curioso etnocentrismo adamítico que aqueja a algunas de sus partes, en concreto a las estadounidenses del “Occupy”, convencidos, algunos de sus manifestantes, de inaugurar la Historia. Pero sí se pueden plantear algunas reflexiones sobre los problemas que pueden tener las alternativas propuestas por dichos movimientos.

\section{Cuestiones finales: los retos}

The next step will be to develop a specific list of goals and demands. As one of the millions of people who are participating in the Occupy Wall

Street movement, I would like to respectfully offer my suggestions of what we can all get behind now to wrestle the control of our country out of the hands of the $1 \%$ and place it squarely with the $99 \%$ majority.

We are but ten weeks old, yet we have already changed the national conversation. This is our moment, the one we've been hoping for, 
waiting for. If it's going to happen it has to happen now. Don't sit this one out. This is the real deal. This is it.

Michael Moore, "Where Does Occupy Wall Street Go From Here”,

Noviembre 2011.

It has been said repeatedly that the Occupy Wall Street movement has no specific agenda. Look at their signs and banners. It is obvious; they want

IN. They no longer want to be excluded, disrespected, unemployed, defrauded, impoverished, betrayed and in big and small ways OUT. They want justice, opportunity and, as the ancient Roman lawyer Marcus

Cicero advocated for, the freedom to participate in power. Ralph Nader, “Let Them In!”, Noviembre 2011.

Thus, the most pressing question facing the Occupy activists is how to craft a unity that respects and celebrates the immense differences among the $99 \%$. How can we learn how to come together? This is something those of the $99 \%$ who are living at Occupy sites can teach us all. How can we come together in a unity that is not simplistic and oppressive, but complex and emancipatory, recognising, in June Jordan's words that "we are the ones we have been waiting for". Angela Davis, “The 99\%: a community of resistance”, Noviembre 2011.

Llegados aquí, la primera pregunta a hacerse es si el movimiento observable es realmente mundial. Obvio que no lo es: no afecta a todos los países (es clamorosa la ausencia africana y del sureste asiático en las movilizaciones del 15-O, es decir, la de los países que Naciones Unidas llama ahora "menos desarrollados”). Además, en los casos en los que sí afecta, no afecta a todos los ciudadanos, lo cual queda particularmente claro cuando las "manifestaciones masivas" son seguidas de triunfos electorales de los objetos del descontento como fue el caso de las "manifestaciones masivas” contra la participación del ejército español en la ocupación de Iraq, seguida de un innegable triunfo del Partido Popular en las inmediatas elecciones municipales ${ }^{20}$. Que los movilizados, aunque masivos, no sean mayoritarios es normal: algunos “tirarán del carro”. Ya no es tan claro que el etnocentrismo de "Occupy” sea funcional para el movimiento en su posible mundialización: muchas de sus voces creen ser los primeros en haber dado el paso mientras otras mantienen el tradicional "aislacionismo" de una determinada tradición política estadounidense. En el peor caso, hay quienes pretenden, desde países centrales, ser los que expliquen al resto del mundo el “¿Qué hacer?”, con el riesgo evidente de neocolonialismo.

Pero lo anterior, igualmente normal (en el sentido de frecuente), tiene consecuencias mayores para la siguiente pregunta, a saber, si estos movimientos proponen un cambio de sistema o un cambio en el sistema. La relativa ausencia de planteamientos sobre países periféricos en los movi- 
mientos de los países centrales, sobre todo en los Estados Unidos, es tan audible como la ausencia de movimientos acordes con aquellos en los países periféricos, aunque los ha habido en algunos y, ciertamente, en América Latina. Los movimientos locales contra un mal o conjunto de males que están a escala mundial tienen poco que hacer, a no ser que se crea en un "efecto mariposa", si no se coordinan a la escala en que se da el problema.

En términos más generales, puede utilizarse la tipología de comportamientos en contextos “anómicos” (con ausencia de normas compartidas) que planteaba el sociólogo estadounidense Robert K. Merton a mitades del siglo pasado ${ }^{21}$, aunque adaptándola a los presentes propósitos argumentales. Lo que aquél pretendía era hacer una tipología de las reacciones sociales ante situaciones de crisis, para lo cual usaba la actitud que se tomase hacia los fines socialmente aceptables y los medios, de modo semejante socialmente aceptados, actitud que podía ser de aceptación (+) o de rechazo (-) según aparece en el siguiente cuadro:

\begin{tabular}{|l|c|c|}
\hline Tipo & Fines & Medios \\
\hline Conformista & $(+)$ & $(+)$ \\
\hline Ritualista & - & + \\
\hline Innovador & + & - \\
\hline Indignado & - & - \\
\hline Revolucionario & $+/-$ & $+/-$ \\
\hline
\end{tabular}

A vista de pájaro, los tipos de reacciones que provocan las crisis actuales se presentan en el cuadro, intuitivamente aunque sin información que lo pruebe, de más frecuentes a menos frecuentes sobre todo en las sociedades de los países centrales. Efectivamente, el conformismo es el comportamiento más frecuente: se observa en aquellos que aceptan tanto las metas del sistema como los medios legítimos para alcanzarlas pero, y esto es muy importante, con independencia de que los logren (bienestar, felicidad, seguridad, identidad, libertad) e incluso con independencia de que consigan acceder a los medios para alcanzarlos (empleo, satisfacción, protección, representación política).

Después vendría el ritualismo de los que aplican mecánicamente los medios aceptados con independencia de que lleven al fin aceptado socialmente. Es propio de las burocracias, incluidas las burocracias políticas, aunque también de la "ciencia normal" en el sentido que le daba Thomas Kuhn.

Los innovadores son los que buscan nuevos medios: puede ser el delito de cuello blanco o el pequeño delito callejero, aunque es obvio que, 
desde el punto de vista de los conformistas, es este último el que más preocupa, aunque el gran delito sea objetivamente más importante, pero no tan visible en la vida cotidiana de quien lee pocos periódicos de "información general”.

Los indignados forman el grupo del que se está hablando en el presente epígrafe: hay un rechazo de los fines y de los medios socialmente aceptados con el particular factor euforizante que produce la inmersión en un grupo de semejantes y que, hay que reconocerlo, puede distorsionar la percepción de los problemas ${ }^{22}$. Pero con una precaución a la hora de incluir a todos los participantes en el movimiento en la misma categoría, lo cual explicaría esa oscilación entre los que proponen cambios en el sistema y los que buscan un cambio de sistema.

En efecto, entre los conformistas y los ritualistas, si no alcanzan las metas sociales, puede producirse y, de hecho, se producen diferentes niveles de frustración dependiendo del tipo de personalidad, del contexto y de la profundidad de la frustración. Los frustrados pueden confundirse con los indignados. Ahora bien, la frustración genera agresividad y la agresividad busca un objeto sobre el que descargarse y puede hacerlo de forma violenta en cuyo caso no estaríamos en el tipo innovador sino en una variante de los dos primeros. Estos agresivos pueden dirigir su agresividad hacia sí mismos (y por eso se explica el aumento de suicidios en los países hasta ahora centrales $^{23}$ ) o hacia fuera, por ejemplo en forma del uso del "Otro" (inmigrante, de diferente religión o sexualidad o supuesta "raza") como objeto de la propia agresividad física (agresiones por parte de las distintas fobias -xenofobia, homofobia etc.-) ${ }^{24}$ o política (auge de los partidos xenófobos en Europa comenzando por Austria y Suiza donde casi alcanzan a un tercio de los votantes) ${ }^{25}$. No vendrá mal, a este respecto, una cita de Bertrand Russel, en este caso, de su "Scila y Caribdis, o comunismo y fascismo" (publicado en 1935 en su colección In Praise of Idleness and Other Essays). Dice así:

El fascismo no es una serie ordenada de opiniones, como el laissezfaire, el socialismo o el comunismo; es, esencialmente, una protesta emocional, en parte de los miembros de la clase media (como los pequeños comerciantes) que sufren las consecuencias del moderno desarrollo económico, en parte de los anárquicos magnates industriales, cuyo amor al poder se ha convertido en megalomanía. Es irracional en el sentido de que no puede conseguir lo que sus defensores desean; no hay filosofía del fascismo, sino solo un psicoanálisis.

El reto es claro: cómo pasar de un comportamiento expresivo de la indignación (comportamiento emocional diferente al de la agresividad) a un comportamiento instrumental (racional con respecto a fines o, por lo menos, racional con respecto a valores según la terminología de Weber) en el que nuevos medios se pongan en práctica para alcanzar fines igualmente nuevos, que es lo que Merton calificaba como revolucionario. Fines probables y medios disponibles para que no se trate de un "wishful thinking", de 
una infantil y freudiana "omnipotencia de las ideas”.

Porque una cosa es apuntar con el dedo, y certeramente, a qué está mal y otra cosa muy diferente es proponer vías viables de cambiarlo y no solo "propuestas" para ver si alguien las recoge, aunque no se excluye lo que Susan George, en su trabajo sobre la OMC, llamó el "efecto Drácula”, a saber, el efecto que se produce cuando determinados asuntos se airean y se sacan a la luz del sol. Es, si se prefiere, el síndrome de "el rey está desnudo”. Se trata, a fin de cuentas, de hacer problemática, para lo cual se precisa mucha información, "esta situación de incompresible respeto por parte del público en general" que es "lo que necesita el financiero para que la democracia no le ate las manos" ( $\mathrm{i} E \mathrm{El}$ texto vuelve a ser el ya citado "El moderno Midas" de Bertrand Russell publicado en 1932!). Si es cierto que "mayo del 68", en su complejidad y heterogeneidad cambió por lo menos la percepción de muchas cosas, los movimientos actuales podrían hacer algo parecido aunque, al carecer de conocimiento de las leyes de la historia, sea difícil pronosticar un resultado u otro.

Pero también es cierto que ahora es más fácil tener un impacto sobre la realidad mundial, dado el profundo desequilibrio en el que se encuentra el sistema mundial y, por tanto, la mayor probabilidad del "efecto mariposa”, efecto producido por pequeños cambios en zonas marginales del sistema. También, probablemente, ahora es más necesario, dadas las crisis contemporáneas. Finalmente, algo se ha podido aprender de los éxitos pasados y también de los errores:

En otras épocas, nos enfrentamos con dureza corrientes que teníamos estrategias diferentes y opuestas para cambiar el mundo. Fuimos derrotados. Hoy nadie puede asegurar que tiene en sus manos el trazado de un camino para llegar a buen puerto. Por eso, sería necesaria mucha más humildad para debatir nuestras diferencias. Para no infligirnos más daños que los que ya nos provoca ese uno por ciento que pretende aplastarnos.

Raúl Zibechi, “La dificultad para manejar nuestros desacuerdos”, Noviembre 2011. 


\section{Notas}

${ }^{1}$ Análisis, evaluaciones y propuestas con autores, entre otros, desde Pablo González Casanova a Immanuel Wallerstein pasando por Osvaldo León, Irene León, Eduardo Tamayo G. y Esther Vivas en Varios Autores, "De indignaciones y alternativas", América Latina en Movimiento, XXV, 471(2011), monográfico. Accesible en http://alainet.org/publica/ alai471w.pdf

${ }^{2}$ http://edant.revistaenie.clarin.com/notas/2009/12/27/_-02107667.htm

${ }^{3}$ Las nuevas tecnologías de la información son un buen ejemplo de "lo bueno en lo malo y lo malo en lo bueno" como titula Johan Galtung. Por un lado, han sido un instrumento de la aceleración de la crisis financiera al permitir 24 horas diarias de negociación en Bolsa y en el mercado de divisas, hecho, en algunos casos, por máquinas conocedoras de las reglas del juego pero que actúan a mucha velocidad y que suponen un porcentaje muy elevado del comercio financiero mundial (Michael Chlistallam, "High-frequency Trading", Deutsche Bank Research, 7 de febrero de 2011, accesible en http://www.dbresearch.de/PROD/ DBR_INTERNET_EN-PROD/PROD0000000000269468.PDF). Pero, por otro, muchos de los movimientos alternativos recientes habrían sido casi imposibles de no ser por dichas tecnologías. Puede verse Yves Gonzalez-Quijano, “Túnez, El Cairo: la revolución árabe y sus orígenes digitales, Awraq, 3 (2011) 87-96; Larbi Chouikha, "Las nuevas tecnologías de la comunicación, vectores de la 'revolución democrática árabe': el caso tunecino”, Íbidem, 97-1

${ }^{4}$ Tom Engelhardt, The United States of Fear Chicago, Haymarket Books 2011.

${ }^{5}$ http://berkeleyearth.org/analysis.php

${ }^{6}$ Joaquín Estefanía, La economía del miedo, Madrid, Galaxia Gutemberg, 2011.

${ }^{7}$ Jörg Friedrichs, "Global energy crunch: How different parts of the world would react to a peak oil scenario”, Energy Policy, vol. 38, n 8 (2010) 4.562-4.569, accesible en http:// www.qeh.ox.ac.uk/pdf/pdf-research/Global\%20Energy\%20Crunch.pdf

${ }^{8}$ Philip B. Smith y Manfred Max-Neef (Economics Unmasked, Totnes, Devon, Green Books, 2011) se refieren a los Estados Unidos como "país en vías de subdesarrollo". Con más razón, puede hablarse en tales términos de los países de la Unión Europea en general y, en particular, de los PIGS, Portugal, Italia, Irlanda, Grecia y España, a los que habría que añadir tal vez Bélgica.

${ }^{9}$ Benjamín Bastida, “Crisis ¿un final por escribir?”, Cuadernos CJ, n 143 (2011); Ignacio Ramonet, “El nuevo 'sistema-mundo'”, Mémoire del luttes, 7 de octubre de 2011, accesible en http://www.medelu.org/El-nuevo-sistema-mundo.

${ }^{10}$ Accesible en http://www.cis.es/cis/opencm/ES/1_encuestas/estudios/ver.jsp? estudio $=11904$

${ }^{11}$ Con la excepción de los BRIC (menos Rusia) en los que se muestra una satisfacción generalizada sobre la dirección que lleva el país y algún que otro resultado discutible (como el de Egipto), los datos del Pew Research Center, para 2011, son accesibles en http:// www.pewglobal.org/database/?indicator=3. Particularmente interesante el Latinbarómetro 
de 2011, accesible en http://www.latinobarometro.org/

${ }^{12}$ De nuevo hay que recurrir a “The Modern Midas” (1932) de Bertrand Russell para darse cuenta de que la situación no es nueva: "En todo el mundo, no solo en Gran Bretaña, los intereses de las finanzas, en los años recientes, han sido opuestos a los intereses públicos en general”.

${ }^{13}$ Sermones radiofónicos accesibles en http://www.ssa.gov/history/fcspeech.html

${ }^{14}$ Deena Stryker, "Why Iceland Should Be in the News, But Is Not", Daily Kos, $1^{\circ}$ de agosto de 2011, accesible en http://www.dailykos.com/story/2011/08/01/1001662/-IcelandsOn-going-Revolution

${ }^{15}$ Véase Ignacio Gutiérrez de Terán e Ignacio Álvarez-Ossorio, Informe sobre las revueltas árabes. Túnez, Egipto, Yemen, Bahrein, Libia y Siria, Madrid, Ediciones de Oriente y Mediterráneo, 2011. "Primavera” puede ser un término engañoso si lo que se quiere decir es que se trata de fenómenos pasajeros, cosa que no parece, por lo menos en la mayoría de los casos. Pero también es cierto que "revuelta” no es “revolución”. Véase Johan Galtung, “The Arab Revolt - What Next?”, Transcend Media Service, 28 de noviembre de 2011, accesible en http://www.transcend.org/tms/2011/11/the-arab-revolt-what-next/.

${ }^{16}$ Pamela Sepulveda, “Student Protests Spread Throughout Region”, InterPress Service, 25 de noviembre de 2011, accesible en http://ipsnews.net/news.asp?idnews=105969

${ }^{17}$ Shane Croucher, “The November 30 Strike: Will Britain Shutdown?”, International Business Times, 25 de noviembre de 2011, accesible en http://uk.ibtimes.com/articles/256042/ 20111125/november-30-strike-britain-shutdown.htm.

${ }^{18}$ Zygmund Bauman afirmó en el diario El País (17 de octubre de 2011) que "El 15-M es emocional, le falta pensamiento" (contraponiendo implícitamente el comportamiento expresivo y el comportamiento instrumental con respecto a fines). Parece un tanto exagerado si se compara con fuentes como Varios Autores, Les veus de les places, Barcelona, Icaria, 2011 donde, además, se encuentran planteamientos que responden a las preguntas de Bauman sobre liderazgo y, sobre todo, sobre la superación del individualismo mediante el sumergimiento en el grupo. Pero es que ambos comportamientos, expresivo e instrumental, no son incompatibles.

${ }^{19}$ Uno de los obstáculos que, por lo menos el movimiento Occupy Wall Street, parece ir superando es la dificultad de reflejar adecuadamente las divisiones de su propia sociedad en términos de género, raza y clase social (varones, blancos y clase media). Véase Sady Doyle, “New Eden, Old Devils”, In These Times, 22 de noviembre de 2011, accesible en http:// www.inthesetimes.com/article/12275/new_eden_old_devils.

${ }^{20}$ En el caso estadounidense, según encuesta de Gallup publicada a 21 de noviembre de 2011, el apoyo a "Occupy”, que dice representar al "99\%”, alcanzaría a un 25 por ciento de los encuestados, el rechazo a un 15 por ciento, pero la indiferencia o el desconocimiento llegaría al 60 por ciento. Los datos pueden verse en http://www.gallup.com/poll/150896/ Support-Occupy-Unchanged-Criticize-Approach.aspx?utm_source=alert\&utm_medium= email\&utm_campaign=syndication\&utm_content=morelink\&utm_term=Politics $\% 20$ $\% 20 U S A$ 
${ }^{21}$ Robert K. Merton, Social Theory and Social Structure, Nueva York, Free Press, 1968 (ed. aumentada).

${ }^{22}$ Es preciso recordar los experimentos de psicología social de Muzafer Sherif y Solomon Asch sobre el peso que tiene el grupo en la percepción de estímulos ambiguos generando conformidad, pero también en conseguir errores de percepción ante estímulos inequívocos.

${ }^{23}$ David Stuckler y otros, "Effects of the 2008 recession on health: a first look at European data”, The Lancet, vol 378, nº 9.786 (2011) 124-125. El gráfico que lo acompaña es bien expresivo del aumento de suicidios en Europa a partir de 2007 rompiendo una tendencia descendente en lo que iba de milenio.

${ }^{24}$ Movimiento contra la Intolerancia, Informe Raxen, número 49, enero-junio 2011 (accesible en http://www.movimientocontralaintolerancia.com/html/raxen/raxen.asp) aunque no algunos epígrafes sean discutibles.

${ }^{25}$ La situación electoral, antes de los comicios suizos de octubre de 2011, venía recogida por Latifa Mouaoued, “Baromètre de l'extrême droite en Europe”, Radio France International, 29 de julio de 2011, accesible en

http://www.rfi.fr/europe/20110728-infographie-extreme-droite-europe . Aunque la UDC suiza vio reducidos sus votos siguió siendo el partido mayoritario. A esto se añade que, en Grecia, Laos - Alarma Ortodoxa Popular, que había llegado en cuarto lugar en las elecciones de 2009, entró a formar parte del gobierno de Papademos, después de la caída de Papandreu. 


\section{Bibliografía}

Chomsky, Noam, "Occupy the future", In These Times, $1^{\circ}$ de noviembre de 2011, accesible en http://www.inthesetimes.com/article/12206/ occupy_the_future/

Gutiérrez de Terán, Ignacio e Ignacio Álvarez-Ossorio (2011), Informe sobre las revueltas árabes. Túnez, Egipto, Yemen, Bahrein, Libia y Siria, Ediciones de Oriente y Mediterráneo, Madrid,.

Merton, Robert K. (1968), Social Theory and Social Structure, Free Press, (ed. aumentada), Nueva York.

Ramonet, Ignacio, “El nuevo 'sistema-mundo'”, Mémoire del luttes, 7 de octubre de 2011, accesible en http://www.medelu.org/El-nuevo-sistema- 